\title{
Vaginal hysterectomy and training: response to Melendez
}

\author{
Michael D. Moen $\cdot$ Holly E. Richter
}

Published online: 20 December 2014

(C) The International Urogynecological Association 2014

Dear Editor,

Dr Melendez points out a key dilemma in current surgical training in gynecology; namely, where should we focus our limited resources and efforts in order to get the best results for our trainees and, ultimately, for their patients [1]? The same dilemma faces practicing gynecology surgeons; namely, what does it take to maintain the skills involved in performing minimally invasive hysterectomy?

Laparoscopic hysterectomy could be applicable for most patients and the existence of simulation laboratories in most training programs makes it an understandable argument to suggest that our efforts should be focused on teaching this technique rather than others.

One of the most difficult aspects of learning laparoscopic skills is the counterintuitive nature of the physical maneuvers required for mastery of this technique. Simulation is now wellestablished as a useful component of training that allows novice surgeons to utilize time outside the operating room to develop these specific basic skills before embarking on their surgical training in the operating room. But there are limits to how much simulation in the laboratory can substitute for learning in the operating room and, ultimately, surgical performance is dependent on performing actual surgical cases. Furthermore, skills learned in the laboratory rapidly deteriorate if performance is not maintained.

Vaginal hysterectomy, on the other hand, is actually the most teachable technique and, because it utilizes traditional

\section{D. Moen $(\bowtie)$}

Chicago Medical School/Rosalind Franklin University, Illinois Urogynecology, Ltd, 1875 Dempster Street, Suite 665, Park Ridge, IL 60068, USA

e-mail: michael.moen@advocatehealth.com

\section{H. E. Richter}

Division of Urogynecology and Pelvic Reconstructive Surgery University of Alabama at Birmingham, Birmingham, AL 35249 , USA instrumentation, the technical aspects (clamping, cutting, suturing, tying) are more easily maintained for the typical general gynecologist [2].

In an ideal world, all gynecology surgeons would have equal expertise in all routes of hysterectomy and would be able to individualize care for each patient based on their specific situation. Unfortunately, it appears that many surgeons today struggle to maintain expertise in even a single hysterectomy technique. Many factors contribute to this situation, but it is clear that the lack of sufficient case volumes, not only during training, but, also once in practice, is a major issue.

It would be an unfortunate situation if we find ourselves subjecting all patients to laparoscopic hysterectomy because that is the only way to ensure sufficient volume to maintain our technical skills. It would be equally unfortunate if a practicing surgeon finds it necessary to periodically spend time in a simulation laboratory to maintain their skills. Either of these options represents a compromise with the better situation of actually performing sufficient numbers of cases to maintain skills in both laparoscopic and vaginal hysterectomy techniques. To suggest abandoning vaginal hysterectomy because we have better simulation laboratories for laparoscopic hysterectomy seems an incomplete solution to the current problem. Better collaboration between high-volume surgeons and their low-volume colleagues working on cases together in the operating room may be more practical and certainly better for our patients than the expectation of residents and practicing surgeons spending more time performing simulations in the laboratory and limiting their experience to laparoscopic approaches only.

\section{References}

1. Melendez J (2014) Vaginal hysterectomy and training. Int Urogynecol J. doi:10.1007/s00192-014-2611-7

2. Moen MD, Richter HE (2014) Vaginal hysterectomy: past, present, and future. Int Urogynecol J 25(9):1161-1165. doi:10.1007/s00192-014-2459-x 